\title{
Clinical Faceoff: Do We Need Special Strategies for Asian Patients with TKA?
}

\author{
Young Hoo Kim MD, Shuichi Matsuda MD, PhD, Tae Kyun Kim MD, PhD
}

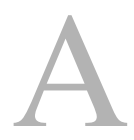
sia, the world's largest continent, is home to 4.4 billion people-a population whose life expectancy is on the rise in many Asian countries. Accordingly, the number of TKAs performed in Asian countries has rapidly increased [14]. Despite the generally successful results of contemporary TKAs, many patients remain dissatisfied with the performance of their replaced knees $[1,8$,

\section{Note from the Editor-in-Chief:}

We are pleased to present to readers of Clinical Orthopaedics and Related Research ${ }^{\mathbb{B}}$ another installment of Clinical Faceoff, $a$ regular feature. This section is a pointcounterpoint discussion between recognized experts in their fields on a controversial topic. We welcome reader feedback on all of our columns and articles; please send your comments to eic@clinorthop.org.

The authors certify that they, or any members of their immediate families, have no funding or commercial associations (consultancies, stock ownership, equity interest, patent/ licensing arrangements, etc.) that might pose a conflict of interest in connection with the submitted article.

All ICMJE Conflict of Interest Forms for authors and Clinical Orthopaedics and Related Research editors and board members are on file with the publication and can be viewed on request.

The opinions expressed are those of the writers and do not reflect the opinion or policy of CORR ${ }^{\mathbb{R}}$ or The Association of Bone and Joint Surgeons ${ }^{\mathbb{R}}$.
20]. The frustration may be even more pronounced among Asian patients, as their functional demands related to a floor-based lifestyle are generally greater than Western patients [7]. Furthermore, if Asian patients have certain demographic and anatomical characteristics known to affect clinical outcomes, special strategies to accom modate the unique nature of Asian patients may be required for preoperative patient counseling, prosthesis design, surgical technique, and postoperative rehabilitation. At the same time, considering the wide variances reported even within the same ethnicity group [16, 18, 25], treating Asian patients undergoing TKA similarly to

Y. H. Kim MD

The Joint Replacement Center, Ewha Womans University Seonam Hospital, Seoul, Republic of Korea

S. Matsuda MD, PhD

Department of Orthopaedic Surgery, Graduate School of Medical Sciences, Kyoto University, Kyoto, Japan

T. K. Kim MD, PhD ( $₫)$

Joint Reconstruction Center, Seoul National University Bundang Hospital, 82 Gumiro 173 Beon-gil, Bundang-gu, Seongnam-si, Gyeonggi-do 13629,

Republic of Korea

e-mail: osktk@snubh.org patients from the West may render better outcomes in function and longevity, because the Western patient population is generally a diverse group, and treatment has evolved to best accommodate this diversity.

To try to bring some clarity to this topic, I have invited two experts on the subject to have a conversation about TKAs in Asian patients: Professor Young Hoo (YH) Kim from the Joint Reconstruction Center of Ewha Womans University Seonam Hospital, in Seoul, South Korea, and Shuichi Matsuda $\mathrm{MD}, \mathrm{PhD}$ from the Department of Orthopaedic Surgery at Kyoto University in Kyoto, Japan. Professor YH Kim is well known for his substantial contribution to the orthopaedic literature in many areas relating to knee arthroplasty. Dr. Matsuda is internationally recognized as a leading knee surgeon with extensive work across basic and clinical research..

Tae Kyun Kim MD, PhD: Let's start with preoperative counseling for Asian patients. Professor Kim, you have done a number of studies in the context of simultaneous bilateral TKA, where the patient serves as his or her own internal control [10, 12]. Many of these found no difference in functional outcomes and longevity by implant 


\section{Clinical Faceoff}

type or surgical technique. As you know, many Asian cultures have functional demands associated with a floor-based lifestyle; how does this influence how you counsel patients in advance of knee replacement?

Young Hoo Kim MD: The goal of TKA is to relieve pain and functional impairment resulting from articular damage. Asian patients demand functionally more than Westerners to accommodate deep squatting and the cross-legged yoga positon associated with a floor-based lifestyle [22, 26]. Prior to surgery, I emphasize to my patients that TKA is a salvage operation to improve pain and function of the arthritic knee joint, and that they might have some functional limitations, including deep squatting and sitting on the floor. Additionally, I advise my patients to sit on a chair as much as possible to avoid deep squatting on the floor. Moreover, I remind my patients that repeated deep squatting and yoga positions may lead to stretching of knee ligaments and could potentially cause long-term instability in the knee.

Shuichi Matsuda MD, PhD: Although I am a bit doubtful of the role repeated deep squatting plays in causing knee joint instability, overall, I agree with Prof. Kim's opinion. Preoperatively, I explain to my patients that TKA can have pain relief and functional improvement, but this surgery will not turn an arthritic knee back into a "young healthy knee." I also inform my patients that they will have some stiffness, and increasing ROM is sometimes difficult to achieve. Therefore, I advise the patients to change from a floor-based lifestyle to chairand bed-based lifestyle.

Dr. TK Kim: Prof. Kim, you have published multiple papers reporting no clinical differences between standard implants and high-flexion knee systems [11, 12] or gender-specific knee implants [10] in Asian patients. To what degree might this be because there simply is not much need for (or benefit from) these implants, and to what degree might your findings be a function of the fact that these were designed initially for (and based on the anatomy of) Western patients?

Prof. YH Kim: Various factors could influence the ROM of the knee after a TKA. Posterior femoral translation has been referred to as a major factor affecting knee flexion. High-flexion TKA was introduced to enhance knee flexion and to facilitate tibiofemoral articulation in Westerners with high flexion. A gender-specific TKA has been introduced to match the three notable anatomic differences in the Western female population: (1) A less prominent anterior condyle, (2) an increased quadriceps angle (Q-angle), and (3) a reduced mediolateral to AP aspect ratio. Although the design characteristics of these high-flexion and gender-specific femoral components are intended to improve clinical outcomes, we and other authors previously reported $[9-11,23,24]$ no difference in the ROM or in the clinical and radiographic results between these TKAs and the standard TKAs. Many studies $[3,5,6]$ have compared the structure of the Asian knee (Japanese, Chinese, Indian, and Korean) to existing Western-designed total knee implant systems. These anthropometric studies have suggested that Westerndesigned knee prostheses may not be suitable for Asian knees. Therefore, some researchers suggested that Asian patients should have specially-designed total knee prosthesis systems. However, many previously published reports [9, 24] suggest that the Western-designed total knee prosthesis functioned equally or even better (including, ROM, knee score, and revision rate) in Asian patients than Westerners. I believe that any type of Western or Asian manufactured TKA can accommodate subtle differences in anthropometric knee anatomy between Asian and Westerners, regardless of different culture, gender, activity level, and ethnicity.

Dr. Matsuda: I think that most of the high-flexion implants are not designed to increase ROM, but rather, are designed to decrease contact stresses in 


\section{Clinical Faceoff}

deep-knee flexion for improving longevity of the implants. I am not surprised that postoperative ROM did not improve with high-flexion impl ants, but their effects should still be assessed by investigating wear-related problems in long-term followup studies.

The advantages of gender-specific implants are more difficult to evaluate. Some research suggests that overhang of the implants are related to knee symptoms [3], but it is not well understood whether or not undersizing can jeopardize the fixation of the implants. Classic scoring systems, ROM, or survival rate would not be suitable for evaluating the benefit of gender-specific implants. Theoretically, gender-specific implants would decrease overhang or undersizing. Perhaps patient-oriented outcome measurements such as the new Knee Society score will show a benefit to using these implants. I agree with Prof. Kim's opinion that Western-manufactured TKA can accommodate subtle differences in anthropometric knee anatomy. Currently, total knee systems cover a wide range of sizes, and the sizing pitch (the increase in the implants' physical dimensions from one size to the next) in most systems is sufficiently small-generally, on the order of a few millimeters-to meet the needs of Asian patients. I believe that sizing pitch is more important than the development of new, ethnic-specific implants.

Dr. TK Kim: Several previous studies reported that Asian patients seeking TKA for their advanced osteoarthritic knees present with different anatomical characteristics and deformity patterns, for example, more severe varus deformity and flexion contracture with yet well-preserved knee flexion [3, 16]. To what degree should the same surgical principles, namely, creation of neutral limb alignment and balanced gaps, be preserved? Or should other approaches be used to try to accommodate functional demands that are specific to the Asian culture, for example, to increase knee flexion degree?

Dr. Matsuda: Generally, the strategies we use to manage joint deformities in Asian patients are not different from those we use when treating other ethnic groups, but we should keep in mind that Asian patients tend to have moresevere bone and joint deformities, in particular large varus-flexion contractures, tibial torsion, and lateral bowing of the femur. In such patients, it is difficult to achieve both neutral alignment and balanced gaps. If normal ligamentous tension is preserved in preoperative knees, recreating pre-OA alignment, such as kinematic alignment, could be one of the reasonable ways to achieve near normal kinematics. In knees with severe varus deformity, however, lateral soft tissues are stretched out [21], and slight undercorrection does not guarantee normal soft-tissue balance. I believe near-neutral alignment is important to avoid abnormal knee motion under the weight-bearing condition. Our recent computer simulation study clearly shows that varus malalignment rather than lateral soft tissue laxity causes lateral lift-off motion [15]. I recommend that we aim for neutral align ment even if this results in some lateral laxity in patients with severe varus knee deformity.

The next question is whether we need special care to achieve neutral alignment for Asian patients. I think many issues should be considered. For example, the tibia has more torsion in Asian people compared to Western population [19]. This would possibly cause malposition of the extramedullary guide. Also, the proximal part of the tibia has greater deformity in Asian people although the midshaft of the tibia is almost straight [27]. We should carefully choose the entry point of the intramedullary guide or set the proximal end of the extramedullary guide for the tibia. Simply choosing the center of the articular surface would result in varus alignment in some patients. Lateral bowing of the femur is another typical deformity in Asian patient [16]. In patients with 


\section{Clinical Faceoff}

severe deformities, we may need additional femoral osteotomy, either simultaneous or staged, along with TKA. Regarding the need for different approaches to obtain more flexion after TKA, although having the ability for high-flexion activities may be important in Asian cultures, I do not think we have to execute different surgical techniques.

Prof. YH Kim: Severe varus knee deformities are associated with contracted medial soft-tissue sleeves and attenuated lateral soft-tissue sleeves. Bony defects in varus knees can be reconstituted by using a bone graft, metal block, or metal sleeve. After a reconstruction of bone defect, contracted soft-tissue sleeves are released until rectangular extension and flexion gap are obtained using a spacer or tensioner, and this will result in attenuated lateral soft-tissue sleeves rega ining their tension. Therefore, we can easily obtain the appropriate $5^{\circ}$ to $6^{\circ}$ valgus alignment of the knee. Wellbalanced medial and lateral soft-tissue sleeves of the knee can minimize lateral lift-off knee motion.

I believe that excessive lateral bowing of femur in Asian patients, particularly in patients with a short stature, is a compensation for their narrow pelvis. Owing to have an excessive lateral bowing, they can avoid impingement of both knees while they are walking. Therefore, an intramedullary femoral jig system can respect their unique anatomy and obtain an appropriate alignment of TKA in patients with excessive lateral bowing.

Many Asian patients' tibiae have double bowing (one at proximal metadiaphyseal junction and another one at distal metadiaphyseal junction) with some tibial torsion. In such patients, the use of an intramedullary tibial guide is somewhat difficult. When using an extramedullary guide, by following the line connecting the medial one-third of tibial tubercle and the second metatarsal bone, appropriate coronal, sagittal and rotational alignments of the tibial component of TKA can be obtained.

In my experience, femoral and/or tibial osteotomy is rarely necessary to correct the deformity of tibia or femur while performing TKA unless they have a malunited fracture of femur or tibia with severe deformity.

Dr. TK Kim: Although Asian patients undergoing TKA are more likely to be older, female, and perhaps less active than those in Western populations, their floor-based lifestyle, which calls for high-flexion activities, may place special demands on patients and implants. How do you consider these factors when counseling patients about postoperative activities?
Dr. Matsuda: Some research suggests that many Asian patients are not interested in returning to sport after knee replacement [2]. However, I recommend patients do light sports activities such as fast walking, swimming, and riding a stationary bicycle. I do believe that physical activity is important for good health, and light participation in sports is fine after knee replacement, as I do not believe these activities jeopardize the longevity of the implants due to their relatively light body weight. Generally, some patients play golf and go hiking, but few play tennis or other high-activity sports in Japan. We should pay more attention to bone quality because our older female patients could potentially have osteoporosis. We tend to cut less bone, using a thinner insert so as not to weaken the bone underneath the implants for such patients. Improving $\mathrm{ROM}$ is an important demand in Asian countries. Although the lifestyle in Japan has become more "Westernized," many Japanese people want to achieve deep flexion for activities of daily living. Surgical techniques that allow for proper patellar tracking and a large enough flexion gap, while also avoiding posterior impingement, are important to achieve deep-knee flexion. Debonding of the anterior flange of the femoral component is another important issue for the patients with high-flexion after TKA. We should 


\section{Clinical Faceoff}

make an adequate bone bed for the anterior flange of the femoral component with proper cementing technique. Furthermore, caution should be taken to avoid anterior notching in knees with severe femoral anterior bowing, particularly when using computer assisted navigation [4, 13, 17]. Postoperative rehabilitation is also impo rtant. Regarding implant design, ideal designs and materials for post-cam mechanisms have not been developed. I allow the patients to perform squats or deep-knee sitting when they have good postoperative ROM. However, in order to avoid high-stress situations, I generally discourage routine kneeling activities for patients with posteriorstabilized implants. I think cruciateretaining implants are more suitable for kneeling activities..

Prof. YH Kim: I encourage all of my patients to be as active as possible after TKA, particularly in walking, bicycling, swimming, and playing golf. I discourage them from getting involved in high-impact sports, heavy lifting, prolonged squatting, mountain hiking, and sitting on the floor in a yoga position. Prolonged sitting on the floor in a yoga position can potentially stretch knee ligaments and may cause late instability of TKAs, particularly in posterior-stabilized TKAs.

\section{References}

1. Bourne RB, Chesworth BM, Davis AM, Mahomed NN, Charron KD. Patient satisfaction after total knee arthroplasty: Who is satisfied and who is not? Clin Orthop Relat Res. 2010;468:57-63.

2. Chang MJ, Kim SH, Kang YG, Chang CB, Kim TK. Activity levels and participation in physical activities by Korean patients following total knee arthroplasty. BMC Musculoskelet Disord. 2014;15:240.

3. Chung BJ, Kang JY, Kang YG, Kim SJ, Kim TK. Clinical implications of femoral anthropometrical features for total knee arthroplasty in Koreans. J Arthroplasty. 2015;30:12201227.

4. Chung BJ, Kang YG, Chang CB, Kim SJ, Kim TK. Differences between sagittal femoral mechanical and distal reference axes should be considered in navigated TKA. Clin Orthop Relat Res. 2009;467:2403-2413.

5. Ha CW, Na SE. The correctness of fit of current total knee prostheses compared with intra-operative anthr opometric measurements in Korean knees. $J$ Bone Joint Surg Br. 2012;94:638-641.

6. Ishimaru M, Hino K, Onishi Y, Iseki Y, Mashima N, Miura H. A threedimensional computed tomography study of distal femoral morphology in Japanese patients: Gender differences and component fit. The Knee. 2014;21:1221-1224.

7. Kim SJ, Bamne A, Song YD, Kang YG, Kim TK. Patients still wish for key improvements after total knee arthroplasty. Knee Surg Relat Res. 2015;27:24-33.

8. Kim TK, Kwon SK, Kang YG, Chang CB, Seong SC. Functional disabilities and satisfaction after total knee arthroplasty in female Asian patients. J Arthroplasty. 2010;25: 458-464 e451-452.

9. Kim YH, Choi Y, Kim JS. Comparison of standard and gender-specific posterior-cruciate-retaining high-flexion total knee replacements: A prospective, randomized study. $J$ Bone Joint Surg Br. 2010;92:639-645.

10. Kim YH, Choi Y, Kim JS. Comparison of a standard and a genderspecific posterior cruciate-substituting high-flexion knee prosthesis: A prospective, randomized, short-term outcome study. J Bone Joint Surg Am. 2010;92:1911-1920.

11. Kim YH, Choi Y, Kwon OR, Kim JS. Functional outcome and range of motion of high-flexion posterior cruciate-retaining and high-flexion posterior cruciate-substituting total knee prostheses. A prospective, randomized study. J Bone Joint Surg Am. 2009;91:753-760.

12. Kim YH, Sohn KS, Kim JS. Range of motion of standard and highflexion posterior stabilized total knee prostheses. A prospective, randomized study. J Bone Joint Surg Am. 2005;87:1470-1475.

13. Ko JH, Han CD, Shin KH, Nguku L, Yang IH, Lee WS, Kim KI, Park KK. Femur bowing could be a risk factor for implant flexion in conventional total knee arthroplasty and 


\section{Clinical Faceoff}

notching in navigated total knee arthroplasty. [Published online ahead of print November 18, 2015]. Knee Surg Sports Traumatol Arthrosc. DOI: 10.1007/s00167-015-3863-6.

14. Koh IJ, Kim TK, Chang CB, Cho HJ, In Y. Trends in use of total knee arthroplasty in Korea from 2001 to 2010. Clin Orthop Relat Res. 2013; 471:1441-1450.

15. Kuriyama $S$, Ishikawa $M$, Nakamura $\mathrm{S}$, Furu M, Ito $\mathrm{H}$, Matsuda S. No condylar lift-off occurs because of excessive lateral soft tissue laxity in neutrally aligned total knee arthroplasty: a computer simulation study. [Published online ahead of print July 4, 2015]. Knee Surg Sports Traumatol Arthrosc. DOI: 10.1007/ s00167-015-3687-4.

16. Lasam MP, Lee KJ, Chang CB, Kang YG, Kim TK. Femoral lateral bowing and varus condylar orientation are prevalent and affect axial alignment of TKA in Koreans. Clin Orthop Relat Res. 2013;471:14721483.

17. Minoda Y, Kobayashi A, Iwaki H, Mitsuhiko I, Kadoya Y, Ohashi H, Takaoka K, Nakamura H. The risk of notching the anterior femoral cortex with the use of navigation systems in total knee arthroplasty. Knee Surg Sports Traumatol Arthrosc. 2010; 18:718-722.

18. Mullaji AB, Marawar SV, Mittal V. A comparison of coronal plane axial femoral relationships in Asian patients with varus osteoarthritic knees and healthy knees. J Arthroplasty. 2009;24:861-867.

19. Nagamine R, Miyanishi K, Miura H, Urabe K, Matsuda S, Iwamoto Y. Medial torsion of the tibia in Japanese patients with osteoarthritis of the knee. Clin Orthop Relat Res. 2003:218-224.

20. Nam D, Nunley RM, Barrack RL. Patient dissatisfaction following total knee replacement: A growing concern? Bone Joint J. 2014;96-B:96100.

21. Okamoto S, Okazaki K, Mitsuyasu $\mathrm{H}$, Matsuda S, Iwamoto Y. Lateral soft tissue laxity increases but medial laxity does not contract with varus deformity in total knee arthroplasty. Clin Orthop Relat Res. 2013;471:1334-1342.

22. Park KK, Shin KS, Chang CB, Kim SJ, Kim TK. Functional disabilities and issues of concern in female Asian patients before TKA. Clin Orthop Relat Res. 2007;461:143-
152.

23. Singh H, Mittal V, Nadkarni B, Agarwal S, Gulati D. Gender-specific high-flexion knee prosthesis in Indian women: A prospective randomized study. J Orthop Surg (Hong Kong). 2012;20:153-156.

24. Song EK, Jung WB, Yoon TR, Park KS, Seo HY, Seon JK. Comparison of outcomes after bilateral simultaneous total knee arthroplasty using gender-specific and unisex knees. J Arthroplasty. 2012; 27:226-231.

25. Tang WM, Zhu YH, Chiu KY. Axial alignment of the lower extremity in Chinese adults. J Bone Joint Surg Am. 2000;82-A:1603-1608.

26. Yoo JH, Chang CB, Kang YG, Kim SJ, Seong SC, Kim TK. Patient expectations of total knee replacement and their association with sociodemographic factors and functional status. J Bone Joint Surg Br. 2011;93:337-344.

27. Yoo JH, Kang YG, Chang CB, Seong SC, Kim TK. The relationship of the medially-offset stem of the tibial component to the medial tibial cortex in total knee replacements in Korean patients. J Bone Joint Surg Br. 2008;90:31-36. 